\title{
Large and small deflections of a cantilever beam
}

Tarsicio Beléndez (1), Cristian Neipp (2) and Augusto Beléndez (2)

(1) Departamento de Ciencia y Tecnología de los Materiales.

Universidad Miguel Hernández de Elche.

Avda. del Ferrocarril, s/n. E-03202. Elche (Alicante). SPAIN

(2) Departamento de Física, Ingeniería de Sistemas y Teoría de la Señal. Universidad de Alicante. Apartado 99. E-03080 Alicante. SPAIN

Corresponding author: A. Beléndez

Phone: +34-6-5903651

Fax: +34-6-5903464

E-mail: a.belendez@ua.es 
BELÉNDEZ, Tarsicio; NEIPP, Cristian; BELÉNDEZ, Augusto. "Large and small deflections of a cantilever beam". European Journal of Physics. Vol. 23, No. 3 (May 2002). ISSN 0143-0807, pp. 371-379

DOI: $10.1088 / 0143-0807 / 23 / 3 / 317$

\begin{abstract}
The classical problem of deflection of a cantilever beam of linear elastic material, under the action of an external vertical concentrated load at the free end, is analyzed. We present the differential equation governing the behaviour of this physical system and show that this equation, although straightforward in appearance, is in fact rather difficult to solve due to the presence of a non-linear term. In this sense, this system is similar to another well known physical system: the simple pendulum. An approximation of the behaviour of a cantilever beam for small deflections was obtained from the equation for large deflections, and we present various numerical results for both cases. Finally, we compared the theoretical results with the experimental results obtained in the laboratory.
\end{abstract}


BELÉNDEZ, Tarsicio; NEIPP, Cristian; BELÉNDEZ, Augusto. "Large and small deflections of a cantilever beam". European J ournal of Physics. Vol. 23, No. 3 (May 2002). ISSN 0143-0807, pp. 371-379

DOI : $10.1088 / 0143-0807 / 23 / 3 / 317$

\section{1.- Introduction}

In this paper we shall analyze an example of a simple physical system, the deflections of a cantilever beam. We shall see that it is not complicated to formulate the equations governing its behaviour or to study it in a physics laboratory at university level. However, a differential equation with a non-linear term is also obtained. Moreover -as occurs with the simple pendulum for small oscillations- [1] when small deflections of the cantilever beam are considered, it is possible to find a simple analytical solution to the problem. In this sense, the study of large and small deflections of a cantilever beam presents a certain analogy with the study of large and small oscillations of a simple pendulum.

The mathematical treatment of the equilibrium of cantilever beams does not involve a great difficulty [2-4]. Nevertheless, unless small deflections are considered, an analytical solution does not exist, since for large deflections a differential equation with a non-linear term must be solved. The problem is said to involve geometrical non-linearity [5, 6]. An excellent treatment of the problem of deflection of a beam, built-it at one end and loaded at the other with a vertical concentrated force, can be found in "The Feynmann Lectures on Physics" [2], as well as in other university textbooks on physics, mechanics and elementary strength of materials. However, in these books the discussion is limited to the consideration of small deflections and they present a formula for the vertical deflection of the end free of the cantilever beam that shows a relation of proportionality between this deflection and the external force applied [2, 4]. The analysis of large deflections of these types of cantilever beams of elastic material can be found in Landau's book on elasticity [5], and the solution in terms of elliptic integrals was obtained by Bisshopp and Drucker [7]. Nevertheless, the developments presented in these last references are difficult for first year university students.

In this paper we analyze the problem of the deflection of a cantilever beam, in the case of both large and small deflections. Firstly, we obtain the differential equation for the deflection curve (elastic curve) in the general case of large deflections, as well as the equations that determine the Cartesian coordinates of each point on the elastic curve. These equations will be solved numerically in an easy way with the aid of the program Mathematica, without using elliptic functions. We obtain the approximation for small deflections from the equations presented for large 
BELÉNDEZ, Tarsicio; NEIPP, Cristian; BELÉNDEZ, Augusto. "Large and small deflections of a cantilever beam". European J ournal of Physics. Vol. 23, No. 3 (May 2002). ISSN 0143-0807, pp. 371-379

DOI : $10.1088 / 0143-0807 / 23 / 3 / 317$

deflections. Various general numerical results are presented and, only by way of example, these are compared with the experimental results obtained in the laboratory.

\section{2.- Theoretical analysis}

We consider a long, thin, cantilever beam of uniform rectangular cross section made of a linear elastic material that is homogeneous and isotropous, in which the law of behaviour of the material is represented by the linear relation:

$$
\sigma=\varepsilon E
$$

that it is known as Hooke's law [2, 8], and where $\sigma$ is the normal stress, $\varepsilon$ is the strain and $E$ is the modulus of elasticity or Young's modulus [2]. The deflection of a cantilever beam is essentially a three dimensional problem. An elastic stretching is one direction is acompanied by a compression in the perpendicular directions: the ration is known as Poisson's ratio. However we can ignore this effect when the length of the beam is larger than the thickness of the perpendicular cross-section and this is shorter than the curvature radius of the beam [2]. In this study, we assume that the beam is non-extensible and strains remain small, and that Bernoulli-Euler's hypothesis is valid, i. e., plane cross-sections which are perpendicular to the neutral axis before deformation remain plane and perpendicular to the neutral axis after deformation. Next, we also assume that the plane-sections do not change their shape or area. Following, for instance, the analysis proposed by Feynmann regarding the study of the deflection of a cantilever beam, it is possible to write the Bernoulli-Euler bending moment-curvature relationship for a uniform-section rectangular beam of linear elastic material as follows [2]:

$$
E I \frac{\mathrm{d} \varphi}{\mathrm{ds}}=M
$$

Where $M$ and $\kappa=\mathrm{d} \varphi / \mathrm{d} s$ are the bending moment and the curvature at any point of the beam respectively, and $I$ is the moment of inertia of the beam cross-section about the neutral axis [2-4]. We will consider the deflections of a cantilever beam subjected to one vertical concentrated load at the free end, by supposing that the deflection due to its self-weight is null. This implies considering a mass-less beam [2]. 
BELÉNDEZ, Tarsicio; NEIPP, Cristian; BELÉNDEZ, Augusto. "Large and small deflections of a cantilever beam". European Journal of Physics. Vol. 23, No. 3 (May 2002). ISSN 0143-0807, pp. 371-379

DOI: $10.1088 / 0143-0807 / 23 / 3 / 317$

\section{1.- Large deflections of a cantilever beam}

Figure 1 shows a cantilever beam of length $L$ with a concentrated load $F$ applied at the free end of the beam. In this figure $\delta_{x}$ and $\delta_{y}$ are the horizontal and vertical displacements at the free end, respectively, and $\varphi_{0}$ takes into account the maximum slope of the beam. We take the origin of the Cartesian coordinate system at the fixed end of the beam and let $(x, y)$ be the coordinates of point $A$, and $s$ the arc length of the beam between the fixed end and point $A$. If we differentiate equation (2) once with respect to $s$, we obtain:

$$
E I \frac{\mathrm{d}^{2} \varphi}{\mathrm{d} s^{2}}=\frac{\mathrm{d} M}{\mathrm{~d} s}
$$

where the bending moment $M$ at a point $A$ with Cartesian coordinates $(x, y)$ is given by the equation (Figure 1):

$$
M(s)=F\left(L-\delta_{x}-x\right)
$$

By differentiating equation (4) once with respect to $s$, taking into account the relation $\cos \varphi=\mathrm{d} x / \mathrm{d} s$ and substituting in equation (3), we obtain the non-linear differential equation that governs the deflections of a cantilever beam made of a linear material under the action of a vertical concentrated load at the free end:

$$
E I \frac{\mathrm{d}^{2} \varphi}{\mathrm{d} s^{2}}+F \cos \varphi=0
$$

Equation (5), although straightforward in appearance, is in fact rather difficult to solve because of the non-linearity inherent in the term $\cos \varphi$. As indicated in the introduction, this situation is similar to that which can be found in the study of the simple pendulum [1]. In order to obtain the solution of equation (5), this equation is multiplied by $\mathrm{d} \varphi / \mathrm{d} s$, so that it becomes:

$$
E I \frac{\mathrm{d} \varphi}{\mathrm{d} s} \frac{\mathrm{d}^{2} \varphi}{\mathrm{d} s^{2}}+F \cos \varphi \frac{\mathrm{d} \varphi}{\mathrm{d} s}=0
$$

which can be written as:

$$
\frac{\mathrm{d}}{\mathrm{d} s}\left[\frac{1}{2} E I\left(\frac{\mathrm{d} \varphi}{\mathrm{d} s}\right)^{2}+F \sin \varphi\right]=0
$$


Equation (7) is immediately integrable taking into account that at the free end $\varphi(L)=$ $\varphi_{0}$, where $\varphi_{0}$ is the unknown slope at the free end of the beam (see Figure 1), and from equations (2) and (4), it follows that $(\mathrm{d} \varphi / \mathrm{d} s)_{s=L}=0$. From equation (7) we can obtain:

$$
\left(\frac{\mathrm{d} \varphi}{\mathrm{d} s}\right)^{2}=\frac{2}{E I}\left(F \sin \varphi_{0}-F \sin \varphi\right)
$$

and by integrating it we can obtain the following equation for the $\operatorname{arc}$ length $s$ as a function of the slope $\varphi$ :

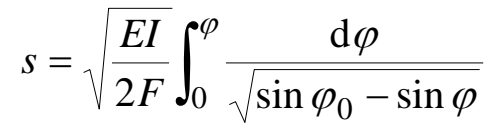

The total length $L$ corresponds to the unknown angle $\varphi_{0}$ at the free end of the beam:

$$
L=\sqrt{\frac{E I}{2 F}} \int_{0}^{\varphi_{0}} \frac{\mathrm{d} \varphi}{\sqrt{\sin \varphi_{0}-\sin \varphi}}
$$

Equation (10) allows us to obtain the angle $\varphi_{0}$ at the free end of the beam as a function of the length, $L$, the modulus of elasticity, $E$, the moment of inertia of the cross-section of the beam, $I$, and the external load, $F$. After obtaining the arc length $s$ as a function of $\varphi$ (equation (9)), and taking into account that $\cos \varphi=\mathrm{d} x / \mathrm{d} s$ and $\sin \varphi=$ $\mathrm{d} y / \mathrm{d} s$, the $x$ and $y$ coordinates of the horizontal and vertical deflection at any point along the neutral axis of the cantilever beam are found as follows:

$$
\begin{aligned}
& x=\sqrt{\frac{2 E I}{F}\left(\sqrt{\sin \varphi_{0}}-\sqrt{\left.\sin \varphi_{0}-\sin \varphi\right)}\right.} \\
& y=\sqrt{\frac{E I}{2 F}} \int_{0}^{\varphi} \frac{\sin \varphi \mathrm{d} \varphi}{\sqrt{\sin \varphi_{0}-\sin \varphi}}
\end{aligned}
$$

From Figure 1, it is easy to see that the horizontal and vertical displacements at the free end can be obtained from equations (11) and (12) taking $\varphi=\varphi_{0}$ :

$$
\begin{gathered}
\delta_{x}=L-x\left(\varphi_{0}\right) \\
\delta_{y}=y\left(\varphi_{0}\right)
\end{gathered}
$$


We introduce the non-dimensional load parameter $\alpha$, and the non-dimensional coordinates $\xi$ and $\eta$ defined as follows:

$$
\begin{aligned}
& \alpha=\frac{F L^{2}}{2 E I} \\
& \xi=x / L \quad \eta=y / L
\end{aligned}
$$

as well as the non-dimensional tip deflection ratios:

$$
\beta_{x}=\delta_{x} / L=1-\xi\left(\varphi_{0}\right) \quad \beta_{y}=\delta_{y} / L=\eta\left(\varphi_{0}\right)
$$

Using the parameter $\alpha$, it is possible to obtain a more general view of the results, because cantilever beams with different combinations of $E, I, F$ and $L$ may give the same value of $\alpha$ and, consequently, they would have the same behavior. Taking into account the definitions of $\alpha, \xi$ and $\eta$, equations (10), (11) and (12) can be written as:

$$
\begin{gathered}
\int_{0}^{\varphi_{0}} \frac{\mathrm{d} \varphi}{\sqrt{\sin \varphi_{0}-\sin \varphi}-2 \sqrt{\alpha}=0} \\
\xi=\frac{1}{\sqrt{\alpha}}\left(\sqrt{\sin \varphi_{0}}-\sqrt{\left.\sin \varphi_{0}-\sin \varphi\right)}\right. \\
\eta=\frac{1}{2 \sqrt{\alpha}} \int_{0}^{\varphi} \frac{\sin \varphi \mathrm{d} \varphi}{\sqrt{\sin \varphi_{0}}-\sin \varphi}
\end{gathered}
$$

Equation (18) allows us to obtain $\varphi_{0}$ as a function of the non-dimensional load parameter $\alpha$. However, equations (19) and (20) are elliptic integrals that may be evaluated numerically [7].

\section{2.- Small deflections of a cantilever beam}

We obtain the approximate solution for small deflections of the cantilever beam from the general equations in the previous section instead of formulating the problem again from the beginning, as is done, for instance, in Feynmann's book [2]. In the case of small deflections, the angle $\varphi$ is small for all the points on the 
cantilever beam. If $\varphi$ and $\varphi_{0}$ are sufficiently small, the approximations $\sin \varphi \approx \varphi$ and $\sin \varphi_{0} \approx \varphi_{0}$ may be used in equations (18)-(20). From equation (18), we can write:

$$
\int_{0}^{\varphi_{0}} \frac{\mathrm{d} \varphi}{\sqrt{\varphi_{0}-\varphi}}-2 \sqrt{\alpha}=0
$$

and integrating it, we can easily see that, for small deflections, the value of the angle at the free end of the beam, $\varphi_{0}$, is equal to the load parameter, $\alpha$, that we introduced in the previous section, and taking into account the definition of $\alpha$ (equation (15)):

$$
\varphi_{0}=\frac{F L^{2}}{2 E I}
$$

From equation (22), we can see that if $\alpha$ is sufficiently small, it is possible to consider small deflections for cantilever beams loaded with a vertical concentrated force at the free end. We can conclude that, provided the approximation $\sin \alpha \approx \alpha$ can be made, it is possible to consider small deflections. For small deflections, equation (19) takes the form:

$$
\xi=1-\sqrt{1-\frac{\varphi}{\alpha}}
$$

we can see that for $\varphi=\varphi_{0}=\alpha$, we obtain $\xi\left(\varphi_{0}\right)=1$, that is, $x=L$ and then $\delta_{x}=0$. This implies that under the hypothesis of small deflections, there is no horizontal displacement at the free end (Figure 2). From equation (4) it can be deduced that, for small deflections, the bending moment is:

$$
M(s)=F(L-x)
$$

which is the equation Feynmann uses as the starting point in his study [2]. Finally, for small deflections, from equation (20) it is easy to obtain the relation:

$$
\eta=\frac{2}{3}\left[\alpha-(\varphi+2 \alpha) \sqrt{1-\frac{\varphi}{\alpha}}\right]
$$

The vertical displacement at the free, $\beta_{y}$, can be obtained considering $\varphi=\varphi_{0}=\alpha$ in equation (25): 
BELÉNDEZ, Tarsicio; NEIPP, Cristian; BELÉNDEZ, Augusto. "Large and small deflections of a cantilever beam". European Journal of Physics. Vol. 23, No. 3 (May 2002). ISSN 0143-0807, pp. 371-379

DOI: $10.1088 / 0143-0807 / 23 / 3 / 317$

$$
\beta_{y}=\frac{2}{3} \alpha
$$

Using equations (23) and (25), it is possible to write the following non-dimensional equation for the elastic curve of the cantilever beam for small deflections:

$$
\eta=2 \alpha\left(\frac{\xi^{2}}{2}-\frac{\xi^{3}}{6}\right)
$$

It is easy to check that if we substitute the values of $\xi, \eta$ and $\alpha$ from equations (15) and (16) in equation (27),we obtain the equation for the elastic curve for small deflections which appears as equation (38.41) in reference [2].

\section{3.- Numerical results}

As mentioned above, in order to study large deflections of a cantilever beam subjected to a vertical concentrated load at the free end, it is necessary to know the angle $\varphi_{0}$. To do this, it is necessary to solve equation (18) in order to obtain $\varphi_{0}$ as a function of $\alpha$. Instead of writing equations (18) and (20) in terms of elliptic functions, by means of complex changes of variable which give little insight as to the nature of the solutions, it is easier for the students to solve them numerically using one of the packages of commercial software available. Nowadays all university students of science and engineering are familiar with programs such as Mathematica or Matlab, which also have student versions. Taking this into account, we used the Mathematica program, with the aid of the "NIntegrate" command, to solve the improper integrals in equations (18) and (20). Firstly, we integrate equation (18) between 0 and $\varphi_{0}-10^{-12}$ (in order to avoid singularity) varying the values of $\varphi_{0}$ (the incognita). Using the Mathematica program, we obtained the values of $\varphi_{0}$ as a function of $\alpha$. We chose as the error quota a result of equation (18) lower than $10^{-7}$. This is very illustrative for the students, because they see how it is possible to easily obtain the value of the incognita which is implicit in an improper integral. Figure 3 shows the results obtained for $\varphi_{0}$ as a function of $\alpha$. We can see that for low values of the load parameter $(\alpha<0.25)$, the deflections are small $\left(\varphi_{0}<15^{\circ}\right)$. However, for large values, large deflections are obtained ( $\varphi_{0} \approx 50^{\circ}$ for $\alpha \approx 1.25$ ). 
BELÉNDEZ, Tarsicio; NEIPP, Cristian; BELÉNDEZ, Augusto. "Large and small deflections of a cantilever beam". European J ournal of Physics. Vol. 23, No. 3 (May 2002). ISSN 0143-0807, pp. 371-379

DOI : $10.1088 / 0143-0807 / 23 / 3 / 317$

Knowing $\varphi_{0}$ as a function of $\alpha$, it is easy to calculate the horizontal nondimensional tip deflection ratio using equation (17), and integrating equation (18) with the aid of the Mathematica program, the vertical non-dimensional tip deflection ratio can also be calculated. Figures 4 and 5 show the results obtained. In Figure 5 the value of $\beta_{y}$ for small deflections has been included. It can be seen that the deflection $\beta_{y}$, obtained exactly from equation (18), and its approximative value calculated using equation (26) only coincide for small values of the load parameter $\alpha$, that is, for small deflections of the cantilever beam. Finally, in Figure 6 the elastic curves for different values of the load parameter have been plotted.

\section{4.- Experimental results}

In the laboratory it is possible to design simple experiments in order to analyze the deflection of a cantilever beam with a tip load applied at the end free [4]. For example, Figure 7 shows a photograph of a system made up of a steel ruler of rectangular section built-in at one end and loaded at the free end with a mass. The length of the ruler is $L=30 \mathrm{~cm}$ and it has an uniform rectangular cross-section of width $b=3.04 \mathrm{~cm}$ and heigth $h=0.078 \mathrm{~cm}$. The moment of inertia of the cross section is given bay the equation $[2,4,9]$ :

$$
I=\frac{1}{12} b h^{3}
$$

and its value is $I=1.20 \times 10^{-12} \mathrm{~m}^{4}$. The weigth of the ruler is $W=0.554 \mathrm{~N}$ and an external force $F=3.92 \mathrm{~N}$ acts on the free end of the ruler. The Young's modulus of the ruler material was calculated for small deflections and the value obtained was $E=$ $200 \mathrm{GPa}$ [4]. In the absence of an external load ( $F=0)$, the maximum vertical deflection of the ruler, experimentally measured and theoretically calculated as $W L^{3} / 8 E I$ [2, 9], was approximately $7.8 \mathrm{~mm}$. This means that the non-dimensional vertical deflection due to its own weight is approximately 0.026 , which can be disregarded for large external loads. Taking into account the values of $F, L, E$ and $I$ and equation (15), the calculated value for the non-dimensional load parameter is $\alpha=$ 0.735. From equations (16)-(20), we obtain $\varphi_{0}=0.62993 \mathrm{rad}\left(36.09^{\circ}\right), \beta_{x}=0.1047\left(\delta_{x}\right.$ $=3.14 \mathrm{~cm})$ and $\beta_{y}=0.4053\left(\delta_{y}=12.16 \mathrm{~cm}\right)$. Figure 8 shows the experimental elastic 
curve as well as the one calculated with the aid of equations (18)-(20). The difference between both is due to the weight of the ruler itself, which has not been considered in the theoretical treatment.

\section{5.- Conclusions}

We have studied the deflections of a cantilever beam both theoretically and experimentally. Firstly, we obtained the equations corresponding to the general case of large deflections and from these we deduced the equations corresponding to the case of small deflections. We have shown that, although we are dealing with a simple physical system, it is described by a differential equation with a non-linear term. Although the solutions to the elastica equations could be expressed in terms of elliptic functions, it is much more convenient for undergraduate students to use numerical integration as outlined in this paper. Numerical integration was performed using the Mathematica program to obtain the exact solution. Finally, we have shown that the deflections of a cantilever beam may be easily studied with a simple, easy-toassemble, low-cost experiment, which allows us to experimentally study the deflections of cantilever beams by means of a series of simple measurements, such as lengths and masses. 
BELÉNDEZ, Tarsicio; NEIPP, Cristian; BELÉNDEZ, Augusto. "Large and small deflections of a cantilever beam". European Journal of Physics. Vol. 23, No. 3 (May 2002). ISSN 0143-0807, pp. 371-379

DOI: $10.1088 / 0143-0807 / 23 / 3 / 317$

\section{REFERENCES}

[1] Alonso M and Finn E J 1992 Physics (Massachusetts: Addison-Wesley Publishing) Chap. 10

[2] Feynman R, Leighton R B and Sands M 1989 The Feynman Lectures on Physics, Volume II: Mainly Electromagnetism and Matter (Massachusetts: AddisonWesley Publishing) Chap. 38

[3] Timoshenko S P 1983 History of Strength of Materials (New York: Dover Publications)

[4] Beléndez A, Neipp C and Beléndez T 2001 Experimental study of the bending of a cantilever beam Rev. Esp. Fis. 15 (3) $42-5$

[5] Landau L D and Lifshitz E M 1986 Course of Theoretical Physics, Vol. 7: Theory of Elasticity (Oxford: Pergamon Press) Chap. 17

[6] Lee K 2002 Large deflections of cantilever beams of non-linear elastic material under a combined loading Int. J. Non-linear Mech. 37, 439-43

[7] Bisshopp K E and Drucker D C 1945 Large deflections of cantilever beams Quart. Appl. Math. 272-5

[8] McGill D J and King W W 1995 Engineering Mechanics: Statics (Boston: PWS Publishing Company) Chap. 5

[9] Anderson H L, editor-in-chief 1989 Physicist's Desk Reference: The Second Edition of Physics Vade Mecum (New York: American Institute of Physics) p. 36 
BELÉNDEZ, Tarsicio; NEIPP, Cristian; BELÉNDEZ, Augusto. "Large and small deflections of a cantilever beam". European Journal of Physics. Vol. 23, No. 3 (May 2002). ISSN 0143-0807, pp. 371-379

DOI: $10.1088 / 0143-0807 / 23 / 3 / 317$

\section{FIGURE CAPTIONS}

Figure 1.- Cantilever beam loaded with an external vertical concentrated load at the free end and definitions of parameters for large deflections.

Figure 2.- Cantilever beam loaded with an external vertical concentrated load at the free end for small deflections.

Figure 3.- Values obtained from equation (18) for $\varphi_{0}$ as a function of the nondimensional load parameter $\alpha$.

Figure 4.- The horizontal non-dimensional tip deflection ratio, $\beta_{x}$, as a function of the non-dimensional load parameter $\alpha$, for large deflections (for small deflections $\left.\beta_{x}=0\right)$.

Figure 5.- The vertical non-dimensional tip deflection ratio, $\beta_{y}$, as a function of the non-dimensional load parameter $\alpha$, for large and small deflections.

Figure 6.- Elastic curves for different values of the load parameter.

Figure 7.- Photograph of a cantilever beam loaded with an external vertical concentrated load at the free end.

Figure 8.- Experimental and theoretical elastic curves for the ruler analyzed experimentally. 


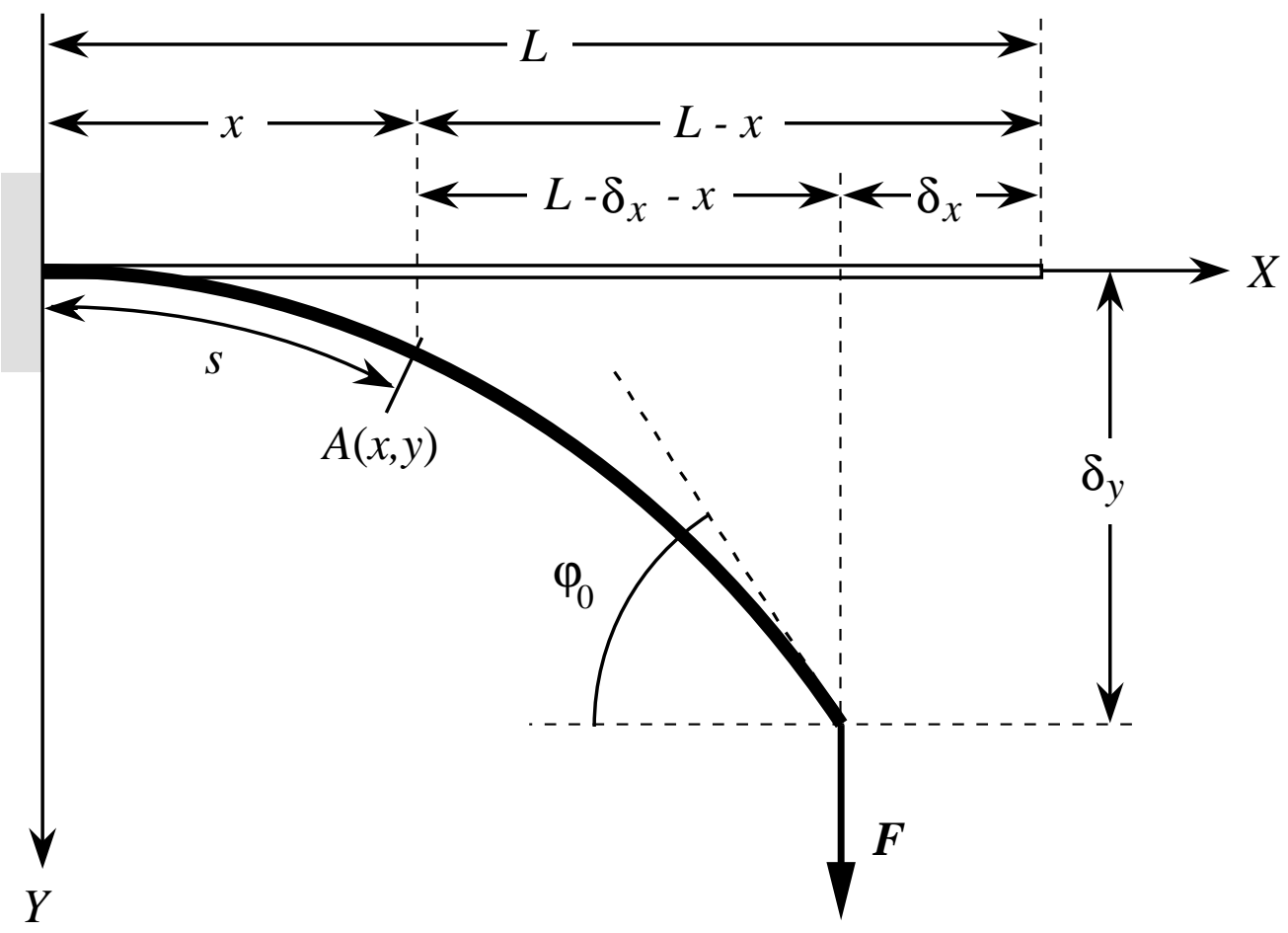

FIGURE 1

Beléndez et al. 


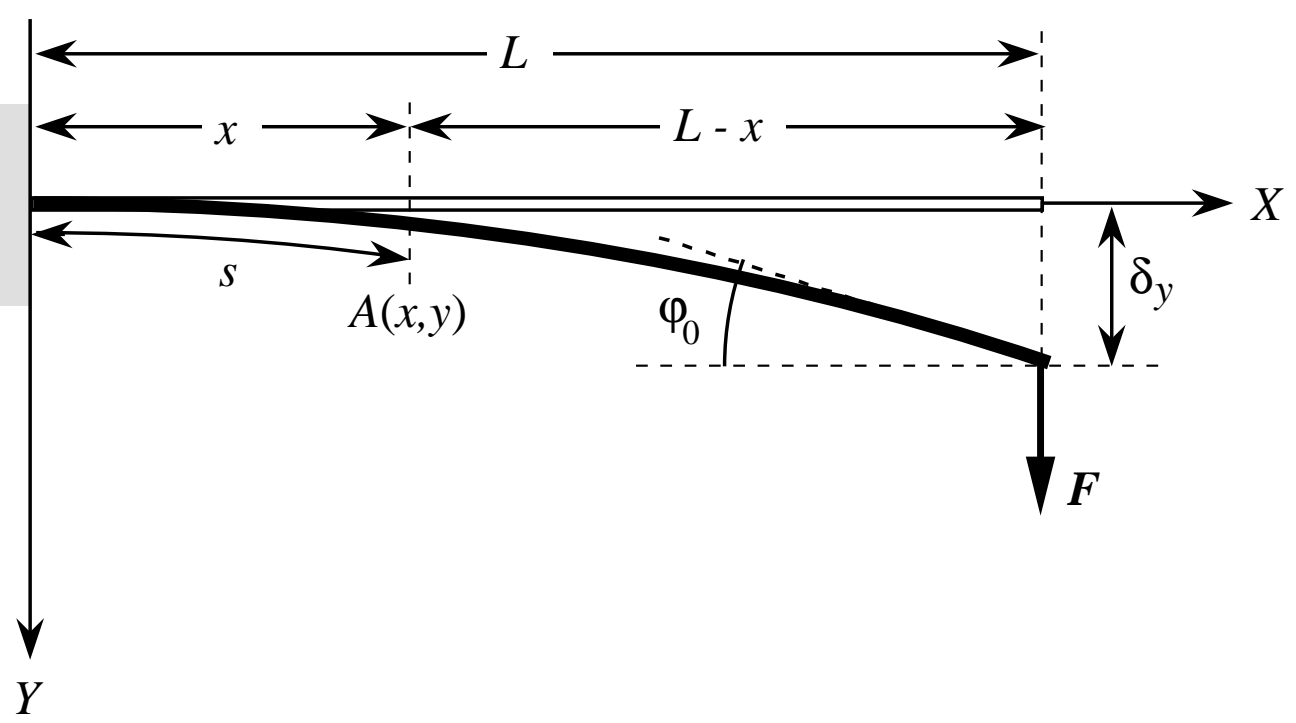

FIGURE 2

Beléndez et al. 


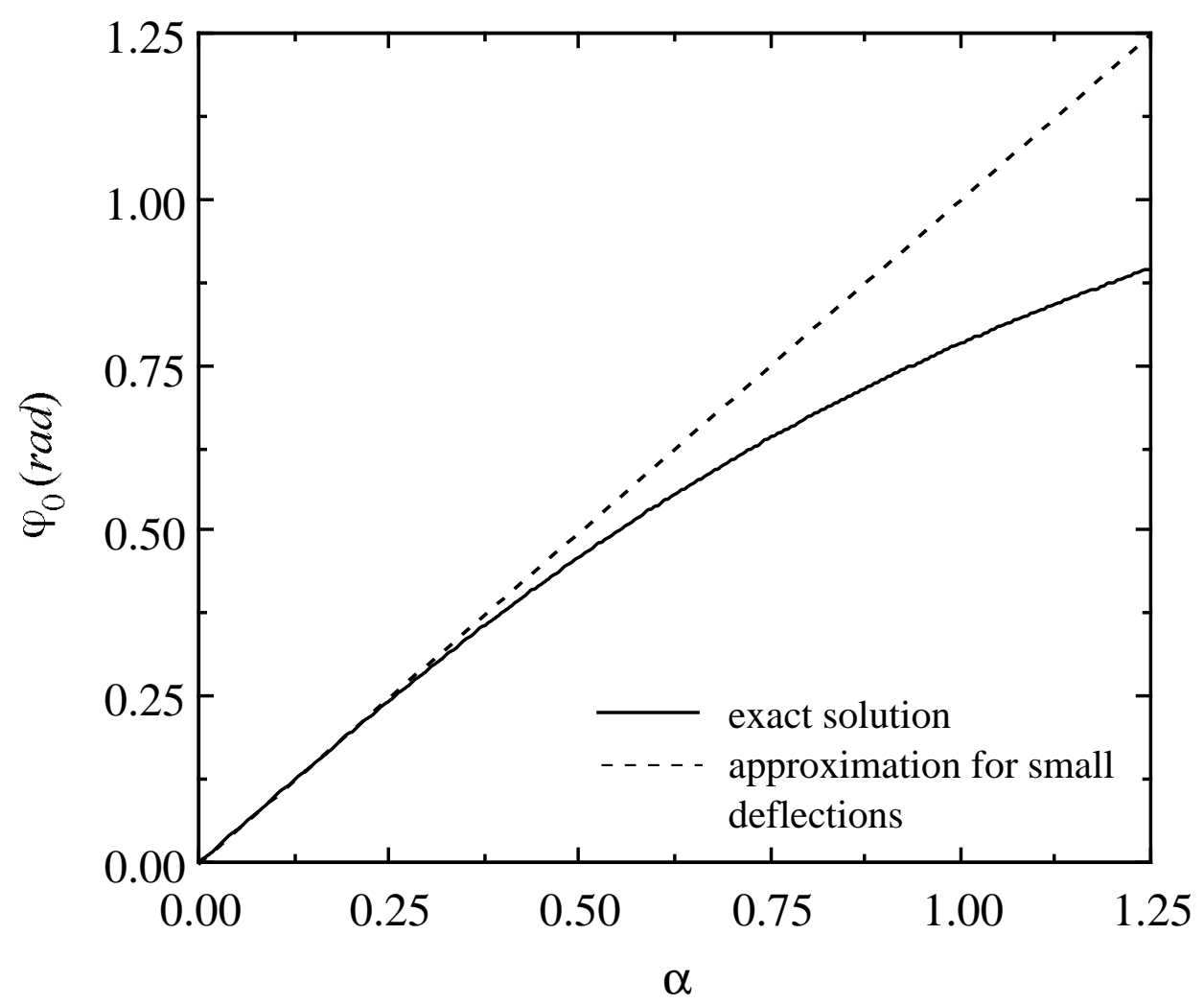

FIGURE 3

Beléndez et al. 


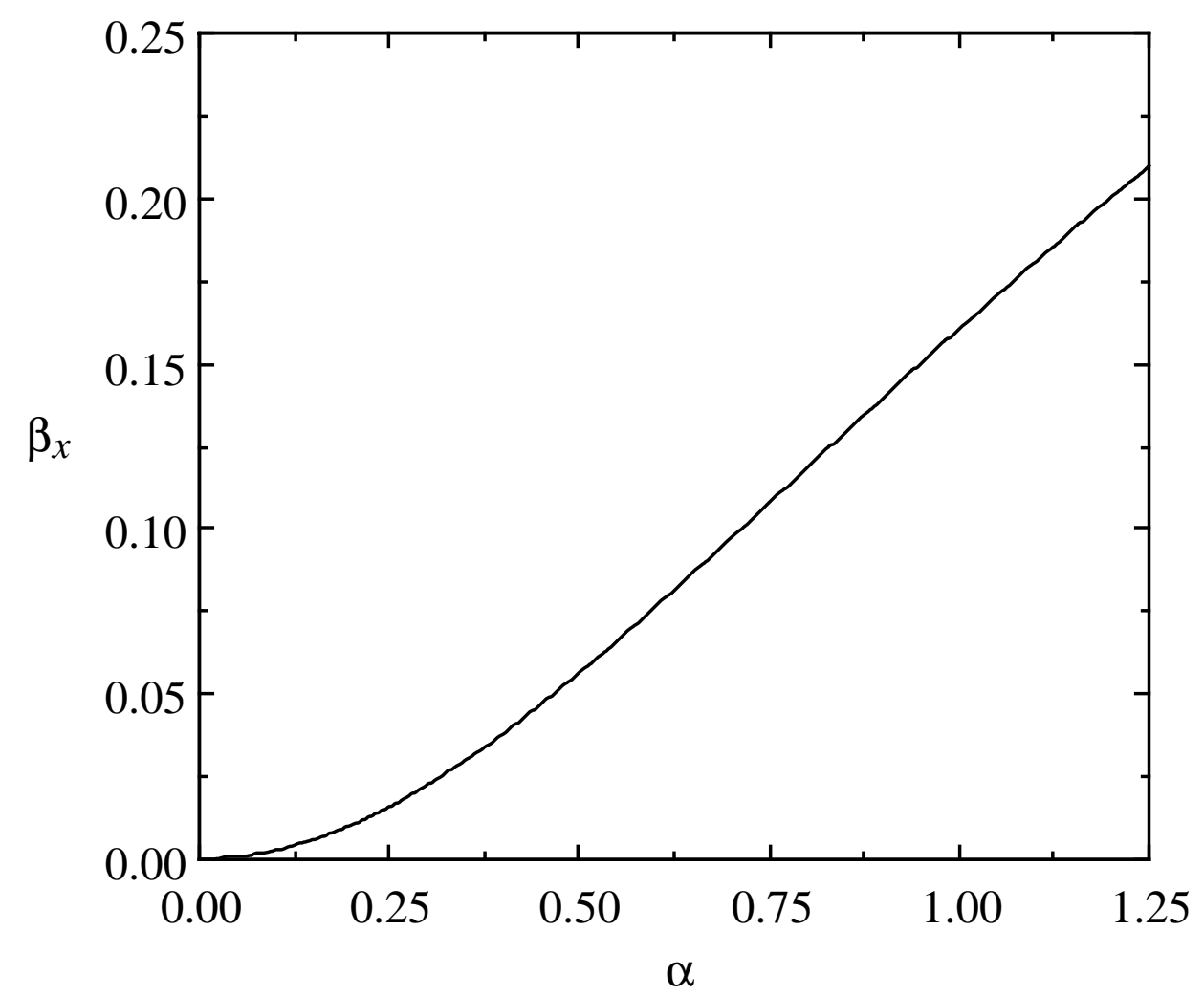

FIGURE 4

Beléndez et al. 


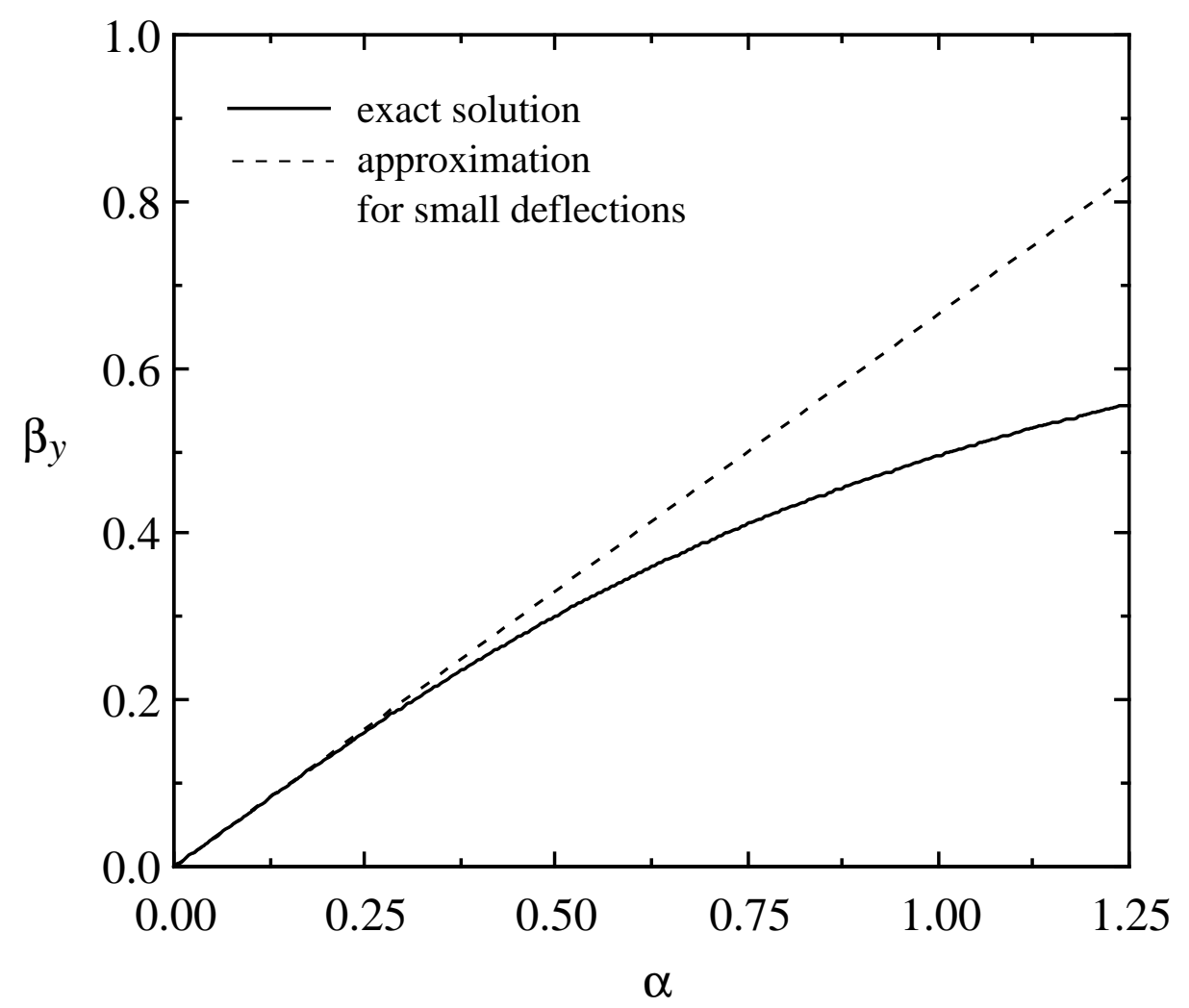

FIGURE 5

Beléndez et al. 

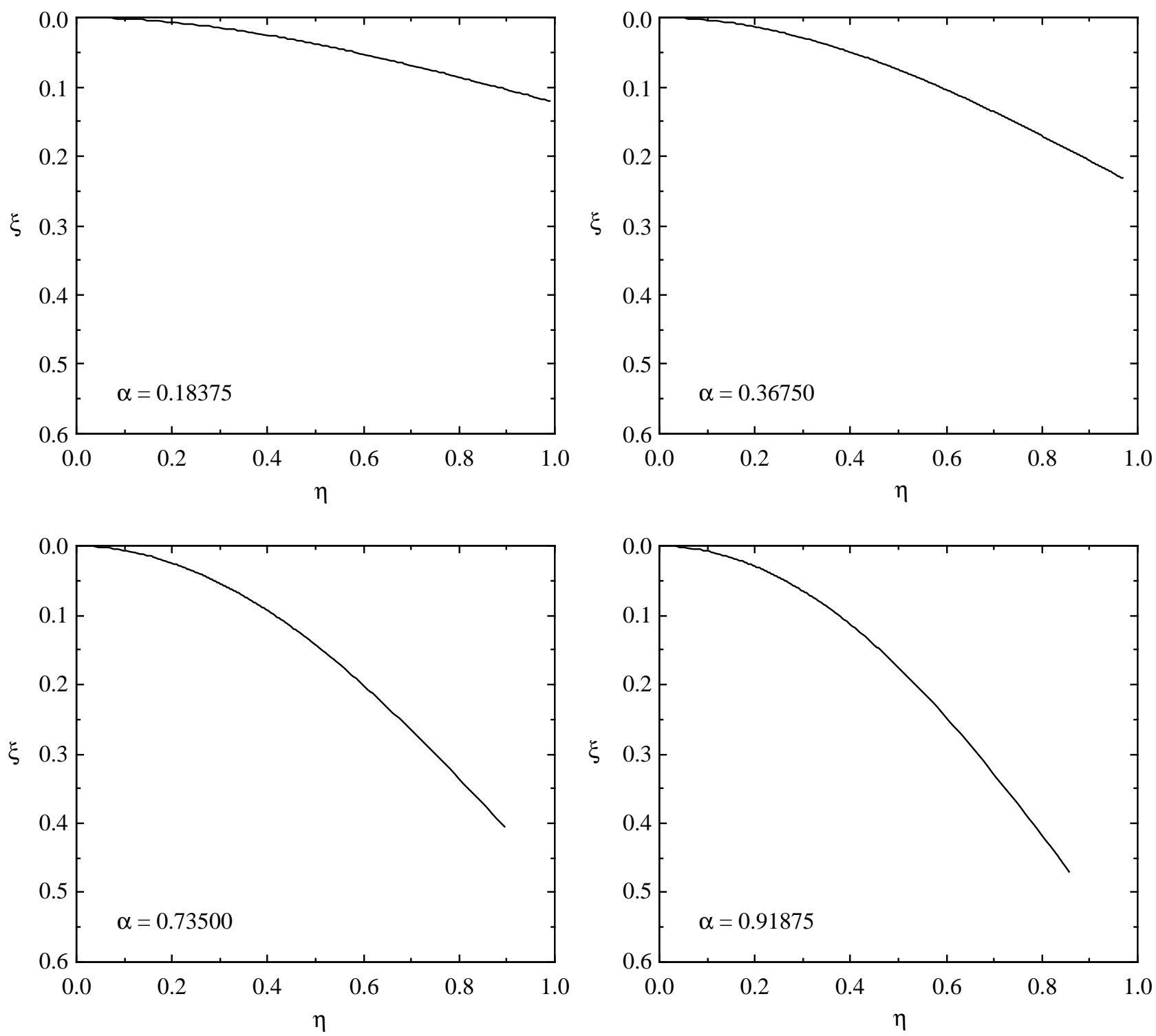

FIGURE 6

Beléndez et al. 


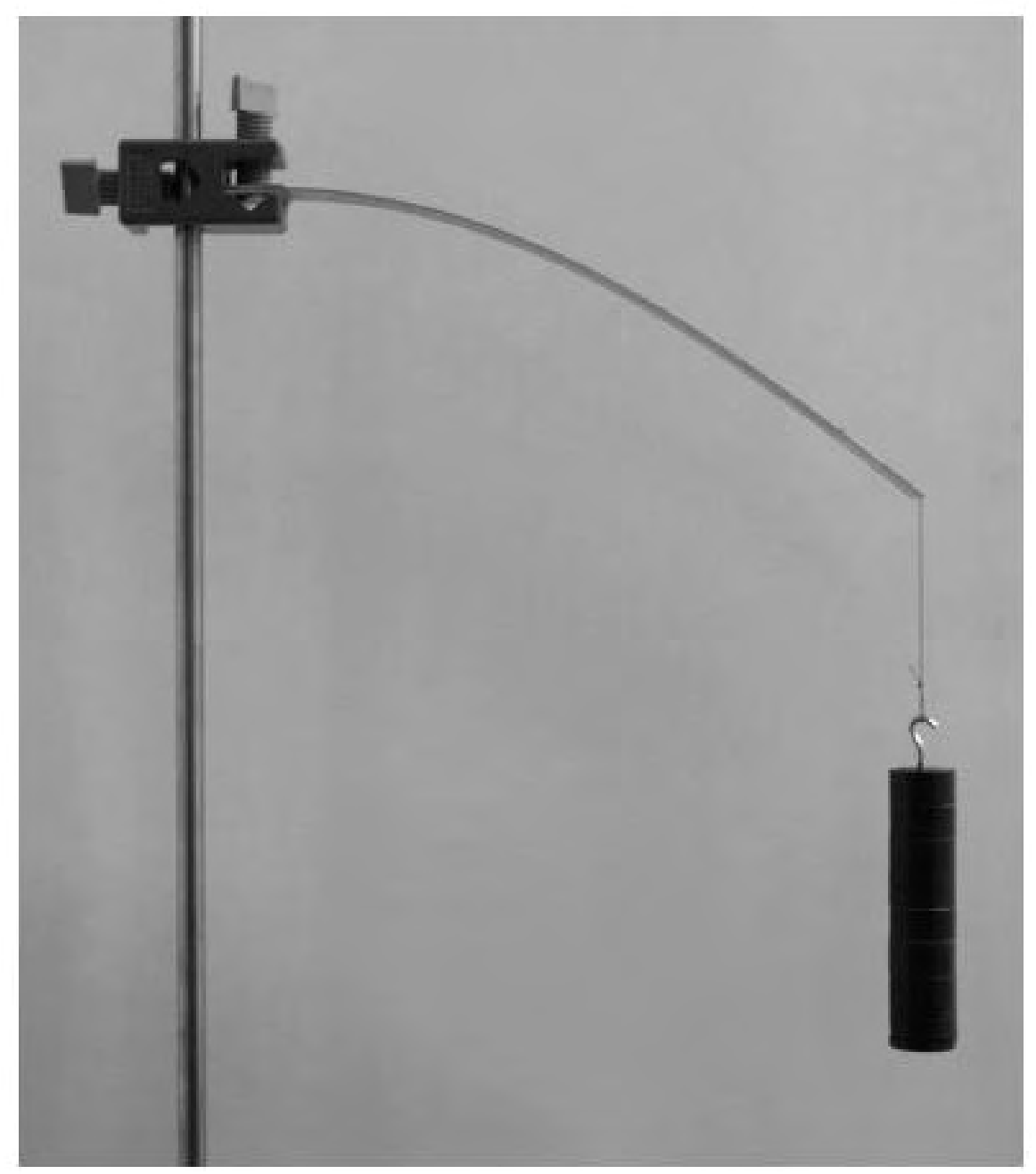

FIGURE 7

Beléndez et al. 


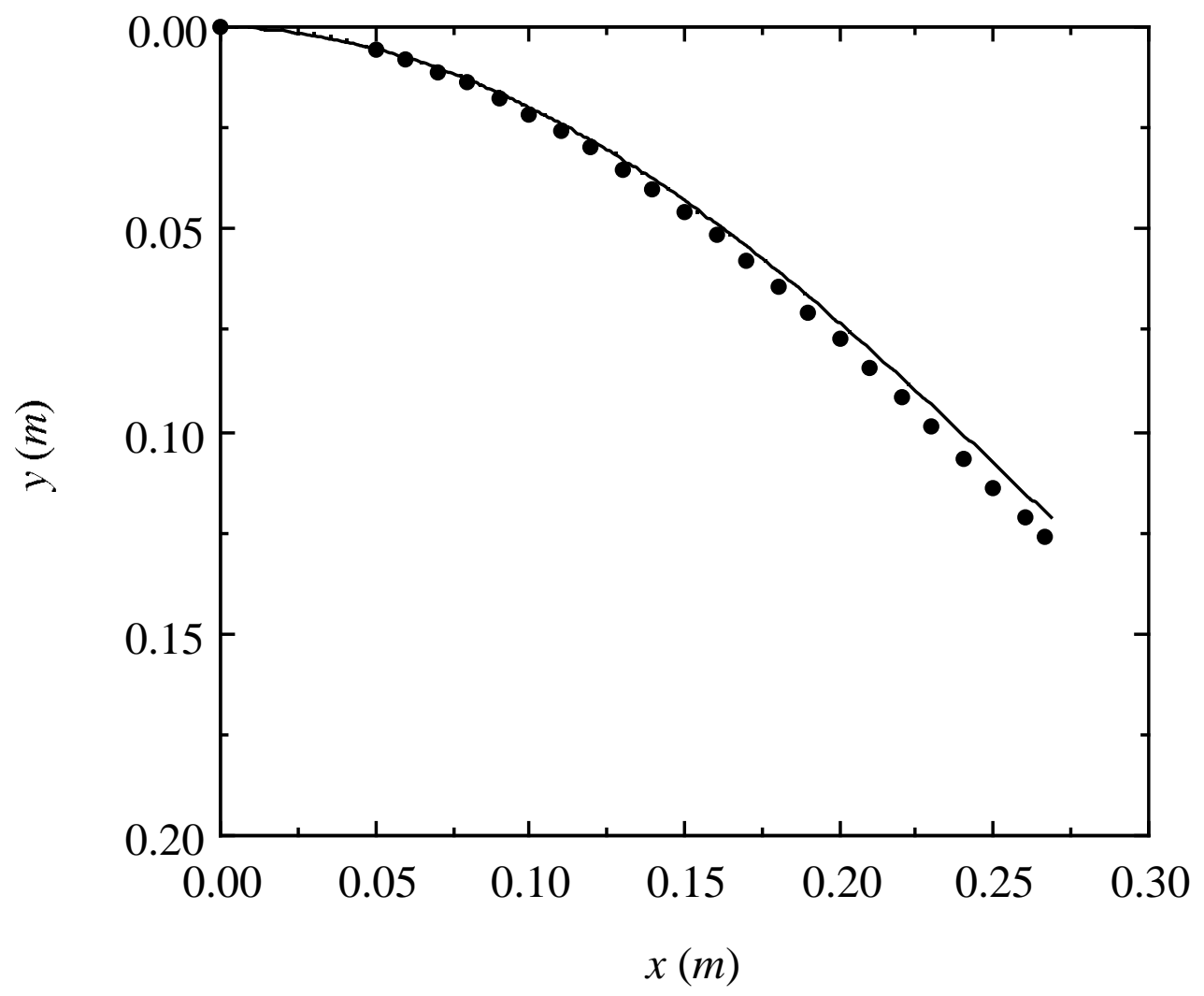

FIGURE 8

Beléndez et al. 